\title{
CPT Category II Codes
}

National Cancer Institute

\section{Source}

National Cancer Institute. CPT Category II Codes. NCI Thesaurus. Code C155824.

A set of supplemental tracking codes that can be used for performance measurement provided by the American Medical Association as part of their Current Procedural Terminology. 